IMECE2005-82790

\title{
DEVELOPMENT OF A PASSENGER WHEEL STANDARD
}

\author{
Jeff Gordon \\ US Department of Transportation \\ Research and Innovative Technology \\ Administration \\ Volpe National Transportation Systems Center \\ Cambridge, MA 02142 USA
}

\author{
Daniel H. Stone \\ Hunter Holiday Consulting, Inc. \\ Pueblo, CO 81003 USA
}

\begin{abstract}
The American Public Transit Association (APTA) is seeking to develop specifications to ensure that wheels used in passenger applications perform safely under the service conditions to which they are exposed. To this end, an approach has been developed which will address this need at two levels. First, a variant on the Association of American Railroads (AAR) S-660 standard [1] is proposed with loading requirements that more realistically represent typical conditions in passenger operations. This is considered a design standard and is to be applied to identify wheel designs not susceptible to fatigue cracking in the wheel plate and hub suitable for use by transit and commuter agencies. Second, an application standard (or more precisely, a recommended practice) has been conceived which is designed to assist transit agencies (or original equipment manufacturers) in the appropriate choice of an "approved" wheel design based on the expected service environment. This technique will identify wheel designs which, under normal operating conditions, should not result in thermal damage to the wheel tread.
\end{abstract}

\section{INTRODUCTION}

The American Public Transit Association (APTA) Passenger Rail Equipment Safety Standards (PRESS) Task Force has been established to develop and maintain standards and recommended practices for passenger rail equipment operating within the general railway system. Many of these standards have been adopted from former AAR standards, support for which AAR has discontinued.

A design standard specifically tailored for passenger applications is needed since the current wheel qualification process embodies several limitations. The most important of these is that the basis for approval consists of an empirical comparison with the results of analyses and historical performance of comparable wheel designs. The revised "S-660 equivalent" design standard is envisioned to be "selfqualifying" in that a review/approval body will not be required and the loading specifications will be more representative of those experienced by passenger wheels. The application standard/recommended practice is an extension of the qualification procedure to actual service conditions. The basic idea is to develop a means by which an operating transit agency may evaluate the likely performance of a particular wheel design based on prescribed operating conditions. This capability serves two purposes. First, wheels appropriate for the agency's current operations may be identified easily. Second, should an agency contemplate changes to its operations (higher speed, heavier vehicles, or other modifications to route characteristics) the impact of such changes on wheel performance may be evaluated.

In combination, the new standard and the companion recommended practice provide a means of compliance with 49CFR 238.231(f) which states that "the operating railroad shall require that the design and operation of the brake system results in wheels that are free of condemnable cracks." While the rule specifically addresses the brake system, this work suggests methods by which a wheel design, appropriate for the envisioned operation, can be chosen to minimize the risk of cracking due to service loading. The paper presents the two approaches. The design standard is currently evolving and few specific details have been resolved. The application standard has progressed somewhat further. The methodology employed in its development and an example application are presented.

\section{EXISTING DESIGN SPECIFICATIONS}

Historically, AAR Standard S-660 has been applied in the industry for the purposes of qualifying wheel designs for use in passenger applications. Standard S-660 has its origins in the 
failure experience with B-28 wheels. Straight-plate B-28 wheels were experiencing a high number of failures while parabolic (curved-plate) B-28 wheels were not experiencing any failures. Finite element analyses of the two designs demonstrated that the curved plate design developed significantly lower stresses than the straight plate design [2]. The S-660 methodology specifies thermal and mechanical loads that are applied to finite element models of the wheel for whose design approval is sought. This practice has served the industry well for several years and has produced a ten-fold reduction in the number of derailments caused by wheel failure from thermal cracks. However, the loading specified in the S660 standard is more relevant to freight operations than passenger applications. Specifically, the thermal loading requirement represents a drag-braking scenario, more common in freight than passenger operations. Further, the current S-660 has several shortcomings:

1. The analysis assumes fully elastic conditions.

2. There is no allowance for the existence of beneficial compressive residual stress that is imparted during manufacture.

3. The basis for approval is an empirical comparison. Approval for use of the wheel in service is granted following favorable comparison (by an independent third party) of results of this analysis with those in a database of previous analysis results for other wheels.

In 2003, the Union Internationale des Chemins de Fer (UIC) introduced a wheel design requirement based on finite element analysis, the results of which are subjected to a fatigue criterion in order to achieve acceptance of the wheel design [3]. As in the current S-660 methodology, a set of thermal and mechanical loads are prescribed and an elastic analysis is conducted. Axle interference (press-fit) stresses are taken into account. Results for all calculation points are plotted on a Haigh (modified Goodman) diagram, the boundaries of which are related to the particular wheel material. If the results at all points lie within the Haigh envelope, the wheel design is deemed acceptable for application in service. The UIC methodology is essentially self-qualifying as the results of the analysis (following the prescribed procedure) determine whether the wheel design will perform safely in service.

\section{PROTOTYPE DESIGN STANDARD}

The eventual design standard is envisioned to be a combination of the current S-660 analysis requirements and the fatigue-based approach of the UIC. The specific details of the loading requirements are still being resolved by the task force developing the standard. The calculation method is intended to provide self-qualification (similar to the UIC approach) in that an independent review and approval body is not required. Execution of the calculation in adherence to the requirements specified in the standard resulting in favorable comparison with the accept/reject criteria will serve as evidence of compliance with the standard.
A three-dimensional finite element calculation will form the basis of the qualification procedure. Initial (asmanufactured) residual stresses present in a new wheel will be included. The precise method by which the heat treatment parameters (which may be proprietary) will be generalized for inclusion in the standard has yet to be determined.

Mechanical and thermal loading requirements will be developed which will likely be defined in the same manner as those currently prescribed by AAR S-660. Load magnitudes have yet to be determined.

The loading conditions will yield three different stress distributions: as-manufactured, mechanical, and thermal. Application of the Sines criterion [4] to the calculated stresses is proposed to infer whether the candidate wheel design is fatigue-prone, by evaluating the following inequality at all material calculation points in the wheel plate and hub:

$$
\frac{1}{3} \sqrt{\left(p_{1}-p_{2}\right)^{2}+\left(p_{2}-p_{3}\right)^{2}+\left(p_{3}-p_{1}\right)^{2}} \geq A-\alpha\left(S_{x}+S_{y}+S_{z}\right)
$$

in which $p_{i}$ are the amplitudes of the alternating principal stresses (due to thermal or contact loading) and $S_{x, y, z}$ are the orthogonal static stresses (the residual stresses from heat treatment). The parameters $A$ and $\alpha$ are temperature-dependent material fatigue properties obtained from testing. A test program was conducted to determine these parameters for AAR Class B wheel steel [5], the results of which yield the values for $A$ and $\alpha$ as a function of temperature shown in Figure 1. A similar test program is underway to obtain these parameters for AAR Class A material and is presented in a companion paper in this conference [6].

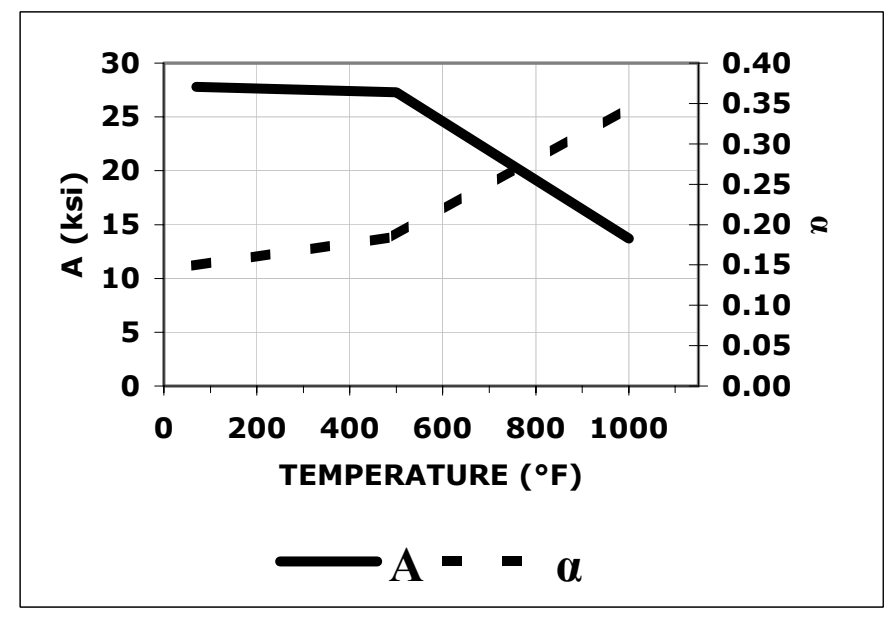

Figure 1. A and $\alpha$ parameters for AAR Class B wheel steel.

\section{PROTOTYPE APPLICATION STANDARD}

Current federal regulations prohibit operation of passenger rail equipment with condemnable wheel tread cracks. To preclude cracking, passenger wheel manufacturing processes include a water-spray rim quench followed by extended high- 
temperature tempering. The heat treatment results in residual hoop compression at the wheel tread to provide resistance to crack formation and growth. Experience has shown that severe tread thermal damage resulting in widespread cracking can occur in spite of the presence of the as-manufactured compressive residual stresses [7]. In passenger applications, frictional heating of the wheel tread during tread braking can result in wheel tread cracking due to a phenomenon known as stress reversal. Rim stress reversal occurs when a shallow layer of material at the wheel tread is rapidly heated. Since the strength properties of the material are vastly diminished at high temperature, the heated layer yields in compression because the cooler bulk of the rim resists the local thermal expansion. When the wheel cools following application of the friction brakes, the circumferential (hoop) stress in the plasticallydeformed material reverses from the as-manufactured residual compression to tension in the heat-affected layer. The presence of the tensile layer exacerbates the formation and growth of tread thermal cracks, negating the benefits of the manufacturing heat treatment.

Therefore, this application standard has been devised to identify combinations of service conditions and wheel designs which are not likely to result in thermal cracking. The Volpe National Transportation Systems Center developed the methodology upon which the application standard is based under a previous Federal Railroad Administration (FRA)funded research program $[8,9]$.

\section{METHODOLOGY}

The prototype recommended practice (RP) is a performance-based approach to the selection of wheels to preclude their premature failure in service. The RP seeks to identify maximum loading conditions for particular wheel designs and AAR material classes above which wheel cracking can be expected to occur. While the performance standard will support the safety goal embodied in the regulations, improved economics may also be realized in that reduced wheel reprofiling (truing) to remove wheel tread thermal cracks will prolong wheel life. The RP can be envisioned as a filter which discriminates between combinations of wheel design and operating parameters which are likely to result in poor wheel performance and those which are not. The RP employs relatively few operating parameters, which are well-known and understood by railroad personnel.

The technique involves finite element analysis of typical wheels used in passenger applications. Results obtained using the 32 " $(81 \mathrm{~cm})$ diameter reverse-dish wheel, the finite element model of which is shown in Figure 2, are presented in this paper. Additional wheel designs to be analyzed include: D36 $(91 \mathrm{~cm})$, E40 $(102 \mathrm{~cm})$, and the Amfleet 36" $(91 \mathrm{~cm})$ wheel.

The first step in the analysis involves estimation of the asmanufactured residual stress distribution in the wheel rim, the procedure for which is documented in its entirety in [7]. Following forming, rim-quenching and tempering, a layer of residual compression approximately 0.75 inch $(3 \mathrm{~cm})$ thick is created at the wheel tread. The model accounts for convection, radiation and the temperature dependence of the thermal and mechanical properties. The analysis predicts maximum residual compression on the order of $30 \mathrm{ksi}(210 \mathrm{MPa})$ at the tread surface. The solid curve in Figure 3 describes the stress distribution in the rim following manufacture.
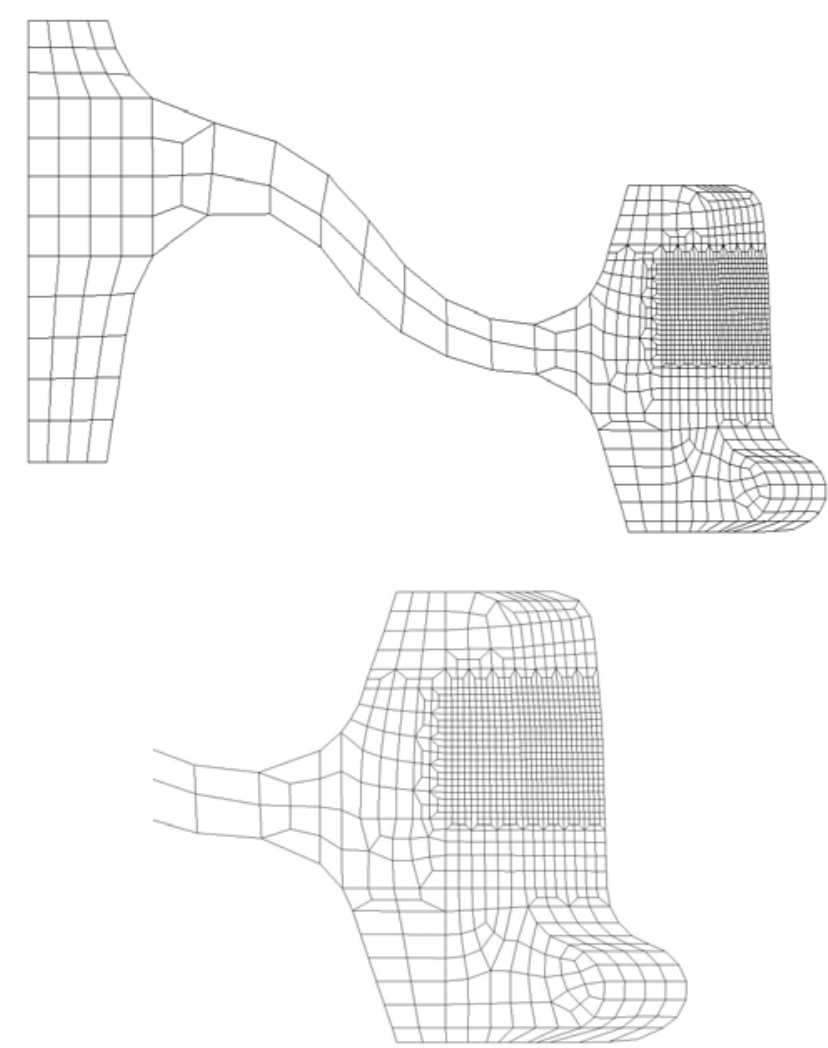

Figure 2. Finite element mesh of 32" reverse-dish wheel and rim detail.

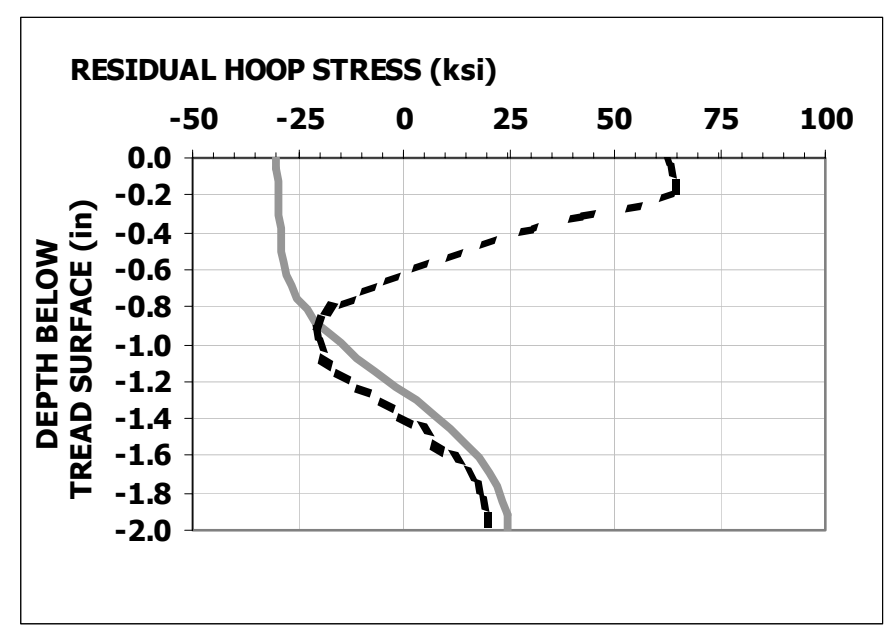

Figure 3. Residual hoop stress along line through rim after manufacture and following simulated service at $100 \mathrm{mph}$. 
Next, the wheel is placed in simulated "service." A matrix of combinations of weight, maximum operating speed and deceleration rate is developed to represent the range of conditions to which the wheel may be exposed. The service simulation variables are presented below:

\begin{tabular}{|c|c|}
\hline WEIGHT: & $\begin{array}{l}\text { 80-180 kips } \\
\text { (36.3-81.6 metric tons) }\end{array}$ \\
\hline OPERATING SPEED: & $\begin{array}{l}80-100 \mathrm{mph} \\
(128-160 \mathrm{kmh})\end{array}$ \\
\hline DECELERATION RATE: & $\begin{array}{l}1.5-3.0 \mathrm{mph} / \mathrm{sec} \\
(2.4-4.8 \mathrm{kmh} / \mathrm{sec})\end{array}$ \\
\hline
\end{tabular}

Each combination of conditions represents an operational braking scenario, each of which is simulated using the technique described in [9]. The residual stress distribution from manufacture is the initial state. The braking simulation is repeated 20 times to establish a stable solution. Braking energy is applied to wheel tread in the form of a uniform heat flux distributed over a region corresponding to the width of the brake shoe. Available estimates in the literature suggest that somewhere between $67 \%$ and $95 \%$ of the frictional heat generated during the stop is absorbed by the wheel. Since many factors influence the heat transfer between the wheel and the brake shoe, an estimate of $80 \%$ (approximately the average) is assumed here. For convenience, the time-dependent heat flux is converted into effective brake power and expressed in units of horsepower (hp; $1 \mathrm{hp}=745.7 \mathrm{~J} / \mathrm{sec}$ ).

Previous studies [9] have shown that contact loading (considered alone and in combination with thermal loads from friction braking) imparts residual hoop compression in the wheel at the tread surface. To avoid computational complexities associated with modeling, contact loading is ignored. This results in some degree of conservatism in the performance-based RP.

The residual stress distribution in the wheel rim after simulated (braking) service is shown in Figure 3 (dashed curve) for a 140-kip (63.5 metric ton) vehicle operating at $100 \mathrm{mph}$ $(160 \mathrm{kmh})$ with a $2 \mathrm{mph} / \mathrm{sec}(3.2 \mathrm{kmh} / \mathrm{sec})$ braking rate. Comparison of the two curves in Figure 3 illustrates the stressreversal concept. After 20 cycles of simulated braking, the asmanufactured residual compression is reversed to tension to a depth of approximately 0.6 inch $(1.5 \mathrm{~cm})$.

The process described above is repeated for 36 combinations of vehicle weight, speed and deceleration rate. The desired "result" for each combination is the depth of stress reversal from compression to tension after the 20 simulated stop braking events. These data are used to infer a brake power limit for the subject wheel for the specified operational braking scenario.

\section{RESULTS}

The results are used to suggest limits on operating conditions, above which thermal damage is expected to occur. The procedures described above are applied to the 32" reverse dish passenger wheel and the results are depicted in Figure 4. Figure 4 illustrates the depth of stress reversal as a function of brake power for all combinations of speed and deceleration rate for the 140-kip vehicle.

For no thermal damage to occur at the wheel tread, the depth of stress reversal must be zero. As this may not prove practical in the eventual application of the RP, a very shallow layer of stress-reversed material $(0.05$ inch, $0.13 \mathrm{~cm})$ is assumed for the purpose of framing the methodology.

The intersections of the vertical dashed line in Figure 4 (at 0.05 ") with the linear fits to the data yield three points which can be used to define a relationship between speed and deceleration rate (for a given vehicle weight) and friction brake power that produce a stress-reversed layer of a prescribed depth.



Figure 4. Stress reversal vs brake power for 9 combinations of speed and deceleration rate (140-kip vehicle).

This process is repeated for a range of vehicle weights, and the results are plotted as shown in Figure 5. Linear fits of these curves have nearly the same slope and are shifted vertically as the assumed vehicle weight is increased. Figure 6 is a polynomial fit of the intercepts of the three curves in Figure 5 and is used to adjust the limiting brake power based on vehicle weight. The fit reaches a maximum at approximately 128 kips (58 metric tons). The data in Figure 5 and Figure 6 can be 
combined to infer the applied brake power $\left(B P_{\text {limit }}\right)$ necessary to result in a small amount of rim stress reversal in terms of the deceleration rate $a$ (in $\mathrm{mph} / \mathrm{sec}$ ) and the vehicle weight $W$ (in kips):

$$
B P_{\text {limit }}=46.4 * a+\left(-0.011 W^{2}+2.8106 W-139.9\right)
$$

for $\mathrm{W} \leq 128 \mathrm{kips}$, and

$B P_{\text {limit }}=46.4 * a+40$

for $\mathrm{W}>128$ kips.

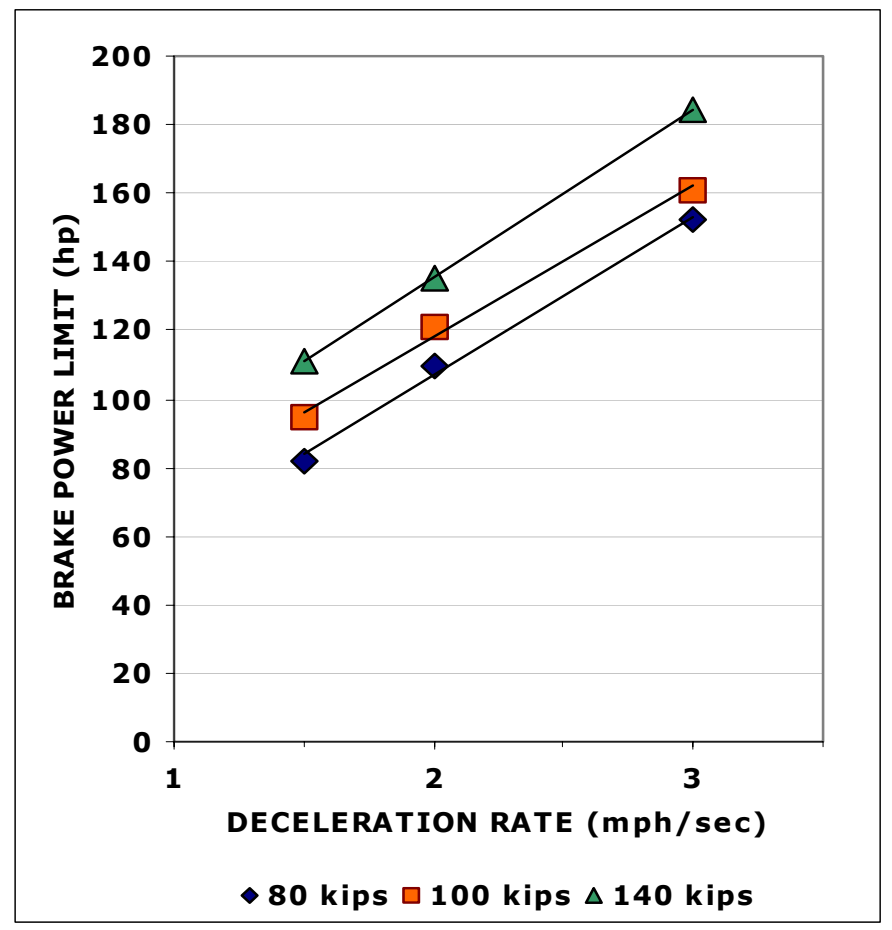

Figure 5. Linear fit to cross-plot of deceleration rate vs brake power for range of vehicle weights.



Figure 6. Polynomial fit to intercepts of curves in Figure 5.

In simple terms, the relationship defined above suggests a brake power limit above which wheel tread thermal damage will occur. The limit is based on deceleration rate and vehicle weight. Maximum operating speed is a secondary factor, since deceleration rate is specified, and essentially defines the time required to stop (or the amount of time that brake power is applied to the wheel tread).

The brake power limit is only applicable to the particular wheel design for which the analysis has been conducted. It can be applied to any set of operating conditions within the range of parameters specified. The procedure described above must be repeated for each wheel design to develop the specific relationship for that design.

To ensure that brake power limits are obtained in a consistent manner, a simple Microsoft Excel spreadsheet is proposed as a tool, shown in Figure 7.

Required input parameters include: vehicle weight, maximum operating speed, fraction of friction braking supplied by tread brakes, and the brake shoe width. The data are entered in the boxes in the upper left portion of the tool. The user may also select whether to include the contribution to braking of the Davis rolling resistance and whether or not to account for nonconstant deceleration rate ("speed taper") by selecting one or both of the check boxes. If speed taper is selected, the user must input sufficient information to define the variable deceleration rates and the speeds with which they are associated, otherwise the user is prompted for a single deceleration rate.

The remainder of the spreadsheet is calculated automatically (wheel load, stop time, and maximum and average brake power). The two plots on the right side of the tool represent the instantaneous friction brake power as a function of time and the deceleration rate as a function of speed. These are useful for visualizing the user input. The tool compares the value calculated for the brake power (172 hp in this example) with values in the "WHEEL DESIGN" table. Table data represent the limiting brake power for each wheel design and material class and are calculated based on user input. The cells turn green indicating the "allowable" wheel design and AAR material classes for the particular operating conditions specified. Inappropriate wheel design/material class combinations are blacked out. The prototype tool contains only one column (for the 32" reverse-dish wheel) currently, but as stated above, the additional wheel designs noted in the table are currently being analyzed to develop the corresponding relationships to complete the table.

\section{LIMITATIONS}

The prototype tool is, at the time of this writing, still evolving. The prototype RP is based on finite element estimates of rim stress reversal. Such models are not readily capable of accounting for certain material characteristics. For example, the distinction between AAR material grades is primarily based on the carbon content of the wheel steel. As carbon content is increased, strength and hardness also increase. Higher strength will result in better performance from the point of view of stress reversal since the calculation 
procedure is based on these properties. Increased hardness typically provides improved wear resistance at the price of reduced fracture toughness, resulting in a more crack-prone wheel depending on the service conditions. This characteristic is not accounted for in the analysis procedure. A surrogate is embodied in the current RP to address this limitation. Brake power limits are developed for wheel designs assuming AAR Class A material properties. The limits are scaled to the other material grades based on the fracture toughness of each relative to that of the Class A material.

Finally, if the wheel is to be subjected to drag braking conditions, then a S-660 analysis must also be completed.

In its present form, the RP addresses a single wheel design. Models of three other designs commonly used by US transit agencies are under development. The same procedure will be applied to develop corresponding brake power limits for inclusion in the selection tool. It is expected that this work will be completed by mid-2005.

Tuning of the RP using operating characteristics and wheel designs of transit agencies is vital. Design/service combinations that currently exist will be evaluated using the tool to confirm that it is capable of distinguishing those that suffer from high thermal cracking rates and those that do not. This process will also identify the degree of conservatism embodied in the tool.

The RP must be approved by the APTA PRESS Task Force. It is expected that it will be presented to APTA for consideration sometime during the third quarter of 2005.

\section{CONCLUSIONS}

Two methods are presented for the evaluation of the fitness-for-service of wheels used in passenger applications. A fatigue-based design standard is being developed to provide assurance that new wheel designs will not experience plate and hub fillet cracking as a result of service loads. An application standard (recommended practice) is also proposed which transit agencies may use to assess the likely performance of wheels in service from the point of view of thermal cracking at the wheel tread. The technique is simple to apply once the analytical procedures have been applied to develop the pertinent data for the respective wheel designs. The methodology described in this paper is under development and its presentation here in its current configuration is intentional in order to solicit constructive suggestions on its improvement. Inclusion of additional wheel designs is underway. Testing of the method using characteristics of operating railroads remains to be conducted. It is envisioned that this effort will identify further limitations or areas of over- or under-conservatism to be addressed. Together, the prototype standards will provide a means of compliance with current FRA regulations.

\section{ACKNOWLEDGMENTS}

The work reported in this paper was carried out under the Rail Equipment Safety program sponsored by the Office of Research and Development, Federal Railroad Administration, under the direction of Ms. Claire L. Orth, Chief, Equipment and Operating Practices Research Division. Ms. Monique Stewart is the Project Manager for the work related to railroad wheels.

The members of the task force developing the APTA application standard and recommended practice include:
Al Bieber
Cameron Lonsdale
James Pilch
Steven Dedmon
Paul Jamieson
Thomas Rusin
Phil Strong
Daniel H. Stone
Jeff Gordon

STV, Inc.
Standard Steel
Standard Steel
Standard Steel
WABCO Transit
Rusin Consulting Corp.
PS Consulting Corp.
Hunter Holiday Consulting
US DOT/Volpe Center

\section{REFERENCES}

[1] Procedure for the Analytic Evaluation of Locomotive and Freight Car Wheel Designs, Association of American Railroads Standard S-660, Adopted 1981, Revised 2004.

[2] Novak, G.E., Greenfield, L.P. and Stone D.H., "Simulated Operating Stresses in 28-in. Dia. Wheels," ASME Paper 75-RT-10, January, 1975.

[3] Technical Approval of Solid Wheels, UIC Code 510-5, February 2003.

[4] Sines, G., "Failure of Materials under Combined Repeated Stresses with Superimposed Static Stresses," NACA Technical Note 3495, November 1955.

[5] McMaster, F. and McKeighan, P., "Fatigue Behavior of Railcar Wheel Steel at Ambient and Elevated Temperature," Southwest Research Institute, San Antonio, TX, August 2003. Report No. DOT/FRA/ORD-03/19. http://www.volpe.dot.gov/sdd/docs /fail/fatgbehavior.pdf

[6] McMaster, F., Robledo, G.P. and Gordon, J.E., "Fatigue Performance of AAR Class A Railroad Wheel Steel at Ambient and Elevated Temperatures," Proc. International Mechanical Engineering Congress and Exhibition, ASME RTD (2005).

[7] Orringer, O., Gray, D.E. and McCown, R.J., "Evaluation of Immediate Actions Taken to Deal with Cracking Problems in Wheels of Rail Commuter Cars," Volpe National Transportation Systems Center, Cambridge, MA, July, 1993. Report No. DOT/FRA/ORD-93/15.

[8] Gordon, J. and Perlman, A.B., "Estimation of Residual Stresses in Railroad Commuter Car Wheels Following Manufacture," Volpe National Transportation Systems Center, Cambridge, MA, June 2003. Report no. DOT/FRA/ORD-03-24.

http://www.volpe.dot.gov/sdd/docs /fail/quench.pdf

[9] Gordon J., Jones, J.A. and Perlman, A.B., "Estimation of Service-Induced Residual Stresses in Railroad Commuter Car Wheels," Proc. International Mechanical Engineering Congress and Exhibition, ASME RTD Vol. 15, 25-32 (1998).

http://www.volpe.dot.gov/sdd/docs/fail/whlperf.pdf 
Maximum vehicle weight, AW3 (Ib)

Maximum operating speed ( $\mathrm{mph}$ )

If disc/tread combination, disc fraction (\%)

Brake shoe width (in)

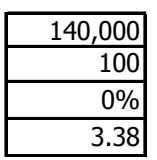

Check to include Davis resistance

Check if variable deceleration rate (speed taper)

Enter number of speed/deceleration rate pairs $(\max 5)$

Complete table to right with up to five (5) speed/deceleration rate pairs

Speed column must be filled in descending

order beginning with max. operating speed.

Fill from top down, with last row at zero speed.

\begin{tabular}{|r|r|}
\hline $\begin{array}{c}\text { SPEED } \\
(\mathrm{mph})\end{array}$ & $\begin{array}{c}\text { DECEL. RATE } \\
(\mathrm{mph} / \mathrm{s})\end{array}$ \\
\hline 100 & 1.70 \\
\hline 50 & 2.00 \\
\hline 0 & 2.00 \\
\hline
\end{tabular}

Maximum static wheel load (lb)

Stop time (sec)

Maximum instantaneous brake power (hp)

Brake power (hp)
17,500
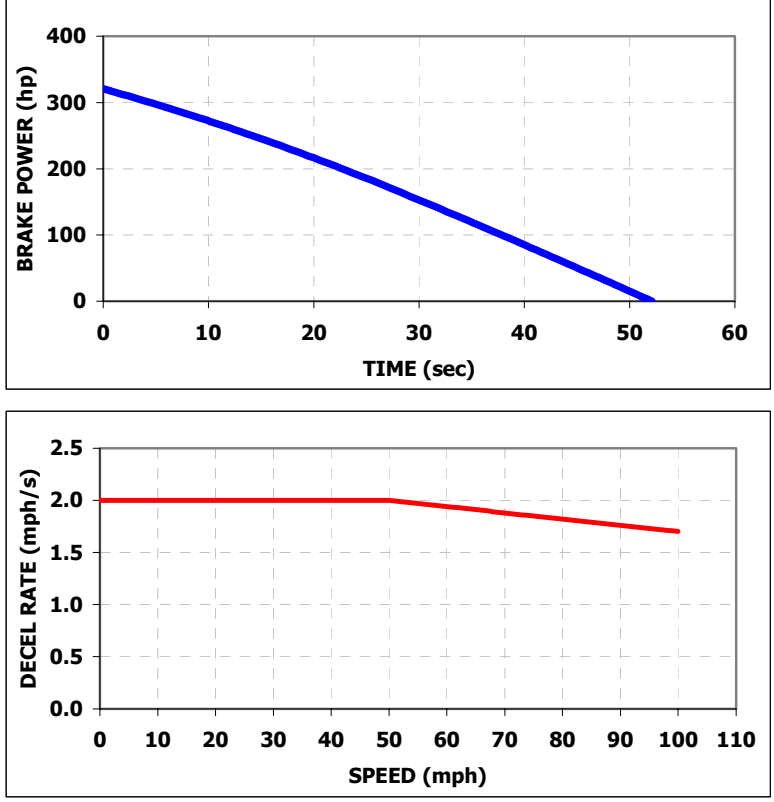

Table entries highlighted in green represent combinations of wheel design and AAR material grade which should not result in thermal cracking for chosen operating conditions.

\begin{tabular}{|c|c|c|c|c|c|}
\hline \begin{tabular}{|c} 
AAR \\
MATERIAL \\
CLASS \\
\end{tabular} & $\begin{array}{c}32 " \\
\text { reverse } \\
\text { dish }\end{array}$ & $\begin{array}{c}\text { Amf } 36 " \\
\text { reverse } \\
\text { dish }\end{array}$ & $\begin{array}{c}\text { D36 } \\
\text { reverse } \\
\text { dish }\end{array}$ & $\begin{array}{l}\text { E40 D } \\
\text { reverse } \\
\text { dish }\end{array}$ & $\begin{array}{c}\text { wheel } \\
\text { design } x\end{array}$ \\
\hline $\mathbf{L}$ & 192 & & & & \\
\hline A & 175 & & & & \\
\hline B & & & & & \\
\hline C & & & & & \\
\hline
\end{tabular}

Figure 7. Prototype wheel selection tool. 